\title{
Regions of interest and parameters for the quantitative analysis of contrast-enhanced ultrasound to evaluate the anti-angiogenic effects of bevacizumab
}

\author{
HUI-PING ZHANG, QIU-SHENG SHI, FAN LI, LONG LIU, MIN BAI, JI-YING GU, YING WU and LIAN-FANG DU \\ Department of Ultrasound, First People's Hospital Affiliated to Shanghai Jiaotong University, Shanghai 200080, P.R. China
}

Received January 22, 2013; Accepted May 2, 2013

DOI: $10.3892 / \mathrm{mmr} .2013 .1499$

\begin{abstract}
The aim of the present study was to identify effective regions of interest (ROIs) and parameters for the quantitative analysis of contrast-enhanced ultrasound (CEUS) to evaluate the anti-angiogenic effects of bevacizumab. Thirty mice were subcutaneously injected with CT26 cells and randomly divided into a bevacizumab-treated (Bev) group and a control group (normal saline-treated). CEUS and quantitative analysis were performed on days 7, 11, 14 and 21 following tumor establishment. $\mathrm{ROI}_{\text {total }}$, which included the whole tumor, and $\mathrm{ROI}_{\text {small }}$, which included the most enhanced part of the tumor, were selected and outlined. Parameters including time to peak (TTP), maximum intensity (Imax) and area under the curve (AUC; in addition to rates of $\mathrm{AUC}_{1}$, $\mathrm{AUC}_{2}, \mathrm{AUC}_{\text {fast }}$ and $\mathrm{AUC}_{\text {slow }}$ ) were recorded. The tumors were resected on day 21 for microvessel density (MVD) counting. Our results showed that the MVD in the Bev group was significantly lower compared with that in the control group (4.09 vs. $6.41 ; \mathrm{P}=0.001)$. Additional parameters of $\mathrm{ROI}_{\text {small }}$ were identified to be significantly different between the two groups, compared with those of $\mathrm{ROI}_{\text {total }}$. No significant differences in TTP and Imax were observed between the two groups at the four time-points examined $(\mathrm{P}>0.05)$. For the AUC parameters in $\mathrm{ROI}_{\text {small }}, \mathrm{AUC}$ and the rates of $\mathrm{AUC}_{2}$, $\mathrm{AUC}_{\text {fast }}$ and $\mathrm{AUC}_{\text {slow }}$ were lower in the Bev group compared with those in the control group on days 7 and $11(\mathrm{P}<0.05)$. These findings indicate that $\mathrm{ROI}_{\text {small }}$ and $\mathrm{AUC}$ parameters in the quantitative analysis of CEUS may be useful for the evaluation of changes in tumor angiogenesis following bevacizumab treatment.
\end{abstract}

Correspondence to: Professor Lian-Fang Du, Department of Ultrasound, First People's Hospital Affiliated to Shanghai Jiaotong University, 85 Wujin Road, Shanghai 200080, P.R. China

E-mail: du_lf@163.com

Key words: contrast-enhanced ultrasound, quantitative analysis, region of interest, angiogenesis, targeted therapy

\section{Introduction}

Anti-angiogenic therapy is one of the most important topics in oncology due to the crucial role of angiogenesis in tumor growth. Among the numerous anti-angiogenic targets, one of the most important is vascular endothelial growth factor (VEGF) (1,2). Bevacizumab is the first humanized anti-VEGF monoclonal antibody to be synthesized based on the theory of angiogenesis and the first clinically available angiogenic inhibitor for metastatic colorectal carcinoma approved by the U.S. Food and Drug Administration (FDA). Bevacizumab exerts its anti-angiogenic effects through the blockade of VEGF signaling by binding to the VEGF-A receptor. Several clinical studies have demonstrated the effects of bevacizumab treatment on advanced colorectal cancer, including improvements to chemotherapy outcomes and survival benefits $(3,4)$. The effective and timely evaluation of the therapeutic effect of treatments is an important issue in imagiology. However, features of the tumor blood supply should be known prior to evaluation of the therapeutic effect. Consequently, novel imaging technologies are urgently required.

Contrast-enhanced ultrasound (CEUS) may be useful for the determination of tumor blood supply. Ultrasound contrast agents (UCAs), including Levovist ${ }^{\circledR}$ and SonoVue ${ }^{\circledR}$, remain exclusively in the vascular compartment and act as blood-pool tracers. Since they have similar diameters to red blood cells, they are ideal tracers for the investigation and evaluation of tissue and tumor perfusion $(5,6)$. The quantitative analysis of CEUS reflects perfusion information within tumors objectively via extracting quantitative information from CEUS imaging (7). Lassau et al (8) evaluated the response to bevacizumab treatment in advanced hepatocellular carcinoma using the quantitative analysis of CEUS and favorable results were obtained; the percentage decrease in several CEUS parameters between days 0 and 3 showed trends toward a correlation with tumor response, progression-free survival and overall survival.

However, quantitative results from CEUS may be affected by a number of factors, including the selection of the region of interest (ROI) and the quantitative parameters, and this should be taken into consideration when interpreting results. A limited number of studies have investigated whether the ROI alone affects results from the quantitative analysis of CEUS. The ROI boundary is usually along the perimeter of 
lesions, or at certain times free from any enhanced area (9). Ignee et al (10) quantitatively analyzed liver parenchyma using CEUS; the size and shape of the ROI were found not to affect the results of the time-intensity curve (TIC). However, a study by Huang-Wei et al (11), which used CEUS to diagnose hepatic focal nodular hyperplasia, demonstrated that the ROIs that included the central region of the lesion more accurately reflected the blood supply in the lesions compared with ROIs that included the whole lesion. Time to peak (TTP) and maximal intensity (Imax) are the most commonly used parameters in the quantitative analysis of CEUS. Area under the curve (AUC) parameters have been increasingly used; however, definitions are not consistent among different studies, which may lead to the acquisition of inconsistent results.

Therefore, in the present study, the quantitative analysis of CEUS was used to investigate changes in the tumor blood supply and determine the effect of bevacizumab on murine colon carcinoma. The aim of the present study was to identify effective ROIs and parameters for the quantitative analysis of CEUS to evaluate the anti-angiogenic effects of bevacizumab.

\section{Materials and methods}

Establishment of animal model.CT26 murine colon carcinoma cells induced by rectal injection of N-nitroso-N-methyl-urethane in BALB/c mice were kindly provided by the Department of Immunology of the Shanghai Second Military University (Shanghai, China). The cells were maintained in RPMI-1640 medium supplemented with $10 \%$ fetal calf serum (FCS). For implantation, single CT26 cell suspensions were harvested and resuspended in RPMI-1640 medium at a density of $2 \times 10^{7}$ cells $/ \mathrm{ml}$. Thirty 4 to 6 -week-old male $\mathrm{BALB} / \mathrm{c}$ nude mice were purchased from the Animal Center of the Chinese Academy of Sciences (Shanghai, China) and housed in specific pathogen-free (SPF) conditions. For each mouse, $0.2 \mathrm{ml}$ of the above-mentioned cell suspension was subcutaneously injected into the hepatic region. All the animal experiments were approved by the Ethics Committee for Animal Research of our institution. The mice were observed daily following tumor establishment. Successful establishment of the tumor model was indicated by the presence of tumors $>5-\mathrm{mm}$.

Grouping and drug administration. Thirty mice were randomly divided into a bevacizumab-treated (Bev) group and a control group ( $\mathrm{n}=15$ mice/group). Mice of the Bev group were intraperitoneally injected with bevacizumab (Roche, Burgess Hill, UK) at a dose of $5 \mathrm{mg} / \mathrm{kg}$ on days 4, 7, 11, 14, 17 and 21 following tumor establishment. Mice in the control group were intraperitoneally injected with normal saline at the same dose and time-points.

Conventional and CEUS examination. Conventional and CEUS examination was performed for all the mice using a Sequoia 512 ultrasound scanner (Siemens Medical Solutions, Mountain View, CA, USA) with a 15L8w transducer (contrast frequency, P7.0 MHz) and Cadence contrast pulse sequencing (CPS) software on days 7, 11, 14 and 21 following injection of the cells. SonoVue microbubbles were used (Bracco,
Milan, Italy), as these are the only microbubbles permitted in China. The microbubbles were prepared according to the manufacturer's instruction prior to CEUS examination. An investigator (with 6 years of experience in small animal CEUS and blinded to grouping information) performed all the ultrasonographic examinations. The mice were placed on the examination table following the induction of anesthesia by an intraperitoneal injection of $50 \mathrm{mg} / \mathrm{kg} 2 \%$ pentobarbital. Ultrasound gel was applied to the tumor to ensure that the distance between the tumor and the probe was $>5 \mathrm{~mm}$, in order to avoid near-field artifacts. Conventional ultrasound was initially used to measure tumor length (L) and width (W). Tumor volume (V) was calculated using the following formula: $\mathrm{V}=\mathrm{LW}^{2} / 2$. A section in which the tumor and mouse liver were able to be simultaneously observed was selected and the CPS mode was activated on the same section. The mechanical index, dynamic range and frame rate were fixed at $0.21,80 \mathrm{~dB}$ and $8 \mathrm{~Hz}$, respectively. SonoVue was prepared (25 $\mu \mathrm{l})$ and administered via the retro-orbital vein as a bolus; a timer was started and the image was observed for $60 \mathrm{sec}$, whilst being recorded simultaneously for offline analysis.

Quantitative analysis of CEUS images. Another investigator (with 5 years of experience in small animal CEUS and also blinded to grouping information) carried out the quantitative analysis of CEUS images using SonoLiver software (TomTec Imaging Systems, Unterschleissheim, Germany) (12). For each dynamic image, $\mathrm{ROI}_{\text {total }}$, which included the whole tumor, and a $5-\mathrm{mm}^{2} \mathrm{ROI}_{\text {refer }}$, which included the normal liver near the tumor, were initially selected and outlined. Following activation of the motion compensation function to minimize the effects of breath movement, the dynamic image was analyzed. Within $\mathrm{ROI}_{\text {total }}$, a $5-\mathrm{mm}^{2} \mathrm{ROI}_{\text {small }}$ was drawn in the region with the highest Imax (Fig. 1). All the raw quantitative information was presented in an excel sheet. Parameters derived from the software in the raw TIC included TTP, Imax, AUC and the rates of $\mathrm{AUC}_{1}, \mathrm{AUC}_{2}, \mathrm{AUC}_{\text {fast }}$ and $\mathrm{AUC}_{\text {slow }}$. TTP and Imax were acquired from the excel sheet and the AUC was calculated using the following formula:

$$
\mathrm{AUC}=\sum_{\mathrm{T} 1}^{\mathrm{T} 2} \text { Intensity }_{\mathrm{t}} / 8
$$

The definitions of these parameters are shown in Fig. 2 and Table I. $\mathrm{AUC}_{\text {fast }}$ and $\mathrm{AUC}_{\text {slow }}$ were calculated according to Schwarz et al (13), where the TIC had a biphasic descending curve, beginning with a fast descending curve and followed by a slow descending curve.

Histological examination. Immediately after CEUS examination on day 21, all the mice were sacrificed and the tumors were removed for immunohistochemical examination using the EnVision method (14). The number of microvessels was determined by CD34 immunostaining with rabbit anti-mouse monoclonal antibody (Abcam, Cambridge, UK) using the Weidner method (15). For each tumor, the numbers of microvessels in 5 hot fields were counted under high-power magnification (x400) and averaged to determine the microvessel density (MVD) parameter. Hot fields were the areas with the highest MVD under low-power magnification (x40), as identified by scanning the section. 
Table I. Definitions for CEUS quantitative parameters.

\begin{tabular}{|c|c|}
\hline Parameter & Definition \\
\hline Imax & Maximum intensity \\
\hline TTP & Time to peak \\
\hline AUC & Area under the whole curve in $60 \mathrm{sec}=\mathrm{AUC}_{1}+\mathrm{AUC}_{2}$ \\
\hline Rate of $\mathrm{AUC}_{1}$ & Area under the ascending curve $\left(\mathrm{AUC}_{1}\right) / \mathrm{TTP}$ \\
\hline Rate of $\mathrm{AUC}_{2}$ & Area under the descending curve $\left(\mathrm{AUC}_{2}=\mathrm{AUC}_{\text {fast }}+\mathrm{AUC}_{\text {slow }}\right) / 60-\mathrm{TTP}$ \\
\hline Rate of $\mathrm{AUC}_{\text {fast }}$ & Area under the fast descending curve $\left(\mathrm{AUC}_{\text {fast }}\right) /(\mathrm{T}-\mathrm{TTP})$ \\
\hline Rate of $\mathrm{AUC}_{\text {slow }}$ & Area under the slow descending curve $\left(\mathrm{AUC}_{\text {slow }}\right) /(60-\mathrm{T})$ \\
\hline
\end{tabular}

CEUS, contrast-enhanced ultrasound.

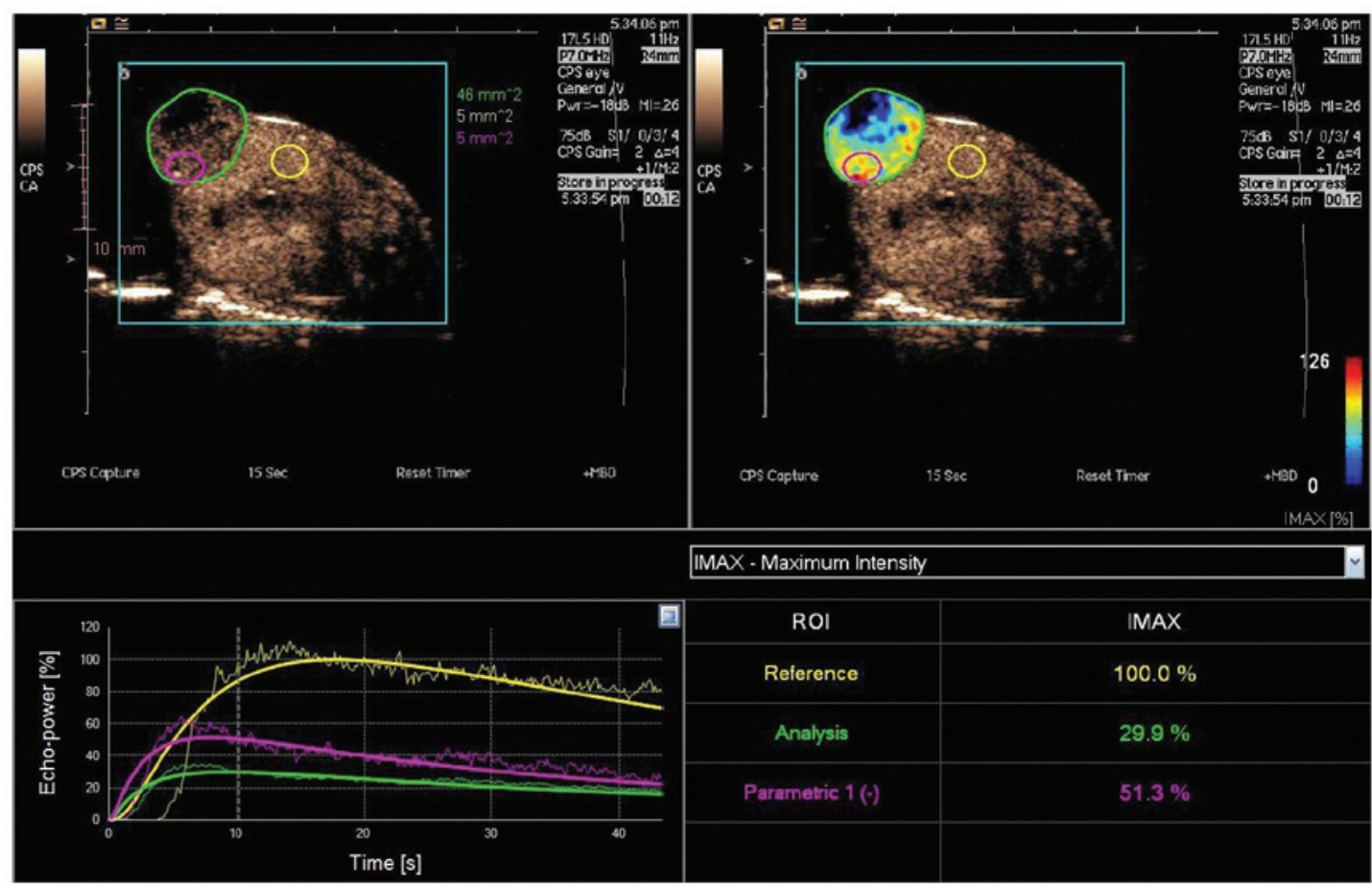

Figure 1. Contrast-enhanced ultrasound (CEUS) image and time-intensity curve for the tumor of a mouse in the control group on day 14 following tumor establishment. The upper left quadrant shows the outlined regions of interest (ROIs) on the image. The green line borders ROI ${ }_{\text {total }}$, which includes the whole tumor. The pink and yellow lines border $\mathrm{ROI}_{\text {small }}$ and $\mathrm{ROI}_{\text {refer }}$, respectively. The upper right quadrant shows $\mathrm{ROI}_{\text {total }}$, which is displayed in color according to maximum

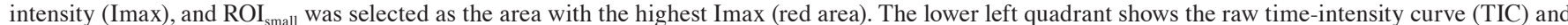
the fitting TIC for the three ROIs, and the lower right quadrant shows the fitting Imax for these three ROIs. ROI ${ }_{\text {refer }}$, includes the normal liver near the tumor.

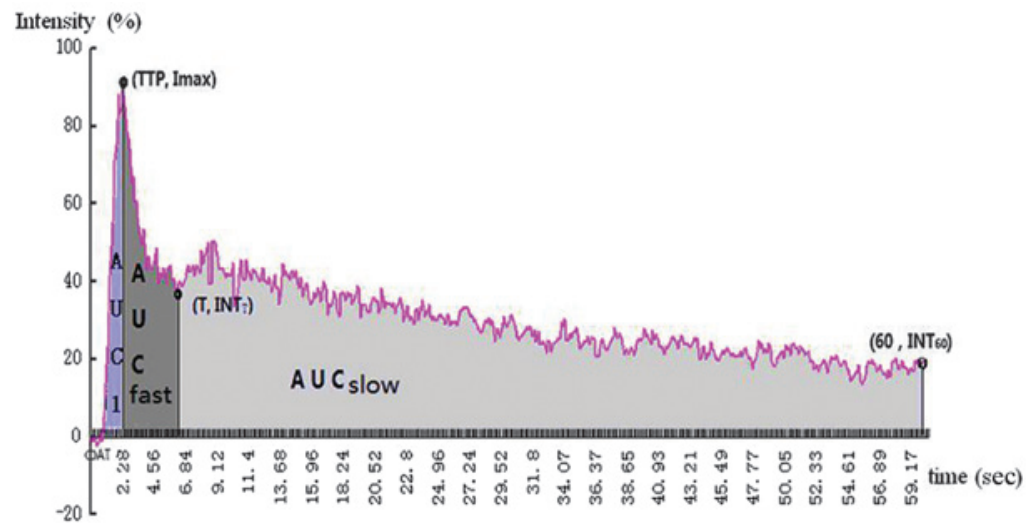

Figure 2. Raw time-intensity curve and quantitative parameters. The coordinate (TTP, Imax) is the cut-off point of the ascending and descending curves. The coordinate (T, INTt) is the cut-off point of the fast and slow descending curves. AUC, area under the curve; TTP, time to peak; Imax, maximum intensity. 


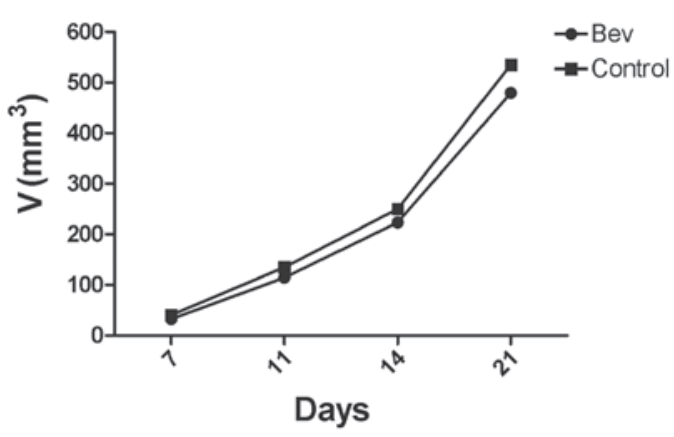

Figure 3. Tumor volume (V) measured by conventional ultrasound in the control and Bev groups at all the four time-points. Tumor volume increased with increasing time in both the control and Bev groups at all the four time-points. However, no significant difference was observed between the two groups at the four time points $(\mathrm{P}>0.05)$. Bev, bevacizumab-treated.
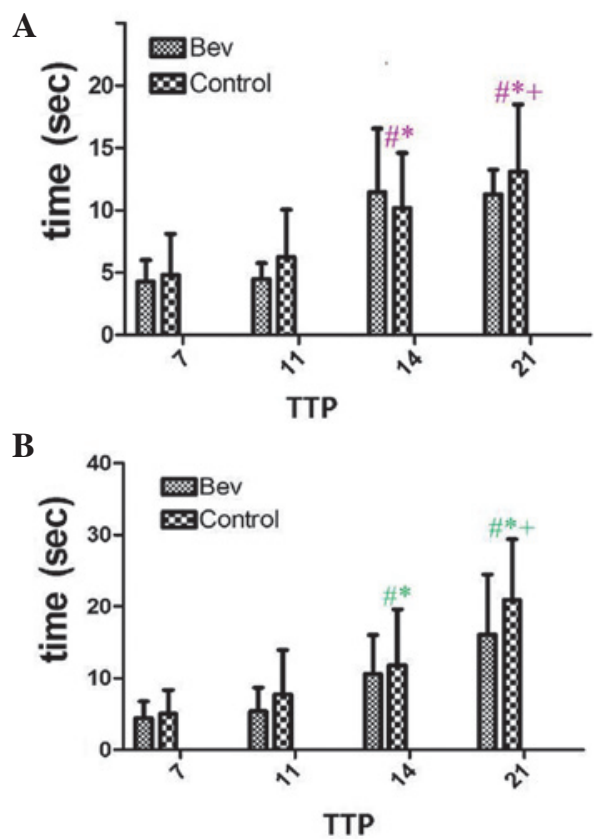

Figure 4. Time to peak (TTP) in the Bev and control groups for (A) ROI small and (B) ROI total . For both $\mathrm{ROI}_{\text {small }}$ and ROI $\mathrm{R}_{\text {total }}$, TTP increased in the control group with increasing time, and no significant difference in TTP was observed between the Bev and control groups at all the four time-points (P $>0.05) .{ }^{*} \mathrm{P}<0.05$ compared with TTP on day $7 ;{ }^{*} \mathrm{P}<0.05$ compared with TTP on day $11 ;{ }^{+} \mathrm{P}<0.05$ compared with TTP on day 14 . Bev, bevacizumab-treated; ROI, region of interest; $\mathrm{ROI}_{\text {total }}$, includes the whole tumor; $\mathrm{ROI}_{\text {small }}$, includes the tumor area with the highest Imax; Imax, maximum intensity.

Statistical analysis. SPSS version 14.0 software (SPSS Inc., Chicago, IL, USA) was used for statistical analysis. The data were expressed as the group mean $\pm \mathrm{SD}$. For parameters that fit the normal distribution, analysis of variance (ANOVA) and independent samples t-tests were used; otherwise, non-parametric testing was used. For all the analyses, $\mathrm{P}<0.05$ was considered to indicate a statistically significant difference.

\section{Results}

Animal model establishment and $V$. The success rate for tumor establishment was $100 \%$ on day 7 following cell administration.


Figure 5. Maximum intensity (Imax) in the Bev and control groups for (A) $\mathrm{ROI}_{\text {small }}$ and (B) $\mathrm{ROI}_{\text {total }}$. Imax in the control group was initially increased, and then decreased in $\mathrm{ROI}_{\text {small }}$, while it was constantly decreased in $\mathrm{ROI}_{\text {total }}$ with increasing time. No significant difference in Imax was observed between the $\mathrm{Bev}$ and control groups at all the four time-points for both $\mathrm{ROI}_{\text {small }}$ and $\mathrm{ROI}_{\text {total }}$ (P>0.05). ${ }^{\text {}} \mathrm{P}<0.05$ compared with Imax on day 7 ; ${ }^{*} \mathrm{P}<0.05$ compared with Imax on day $11 ;{ }^{+} \mathrm{P}<0.05$ compared with Imax on day 14 . Bev, bevacizumab-treated; ROI, region of interest; $\mathrm{ROI}_{\text {total }}$, includes the whole tumor; $\mathrm{ROI}_{\text {small }}$, includes the tumor area with the highest Imax; Imax, maximum intensity.
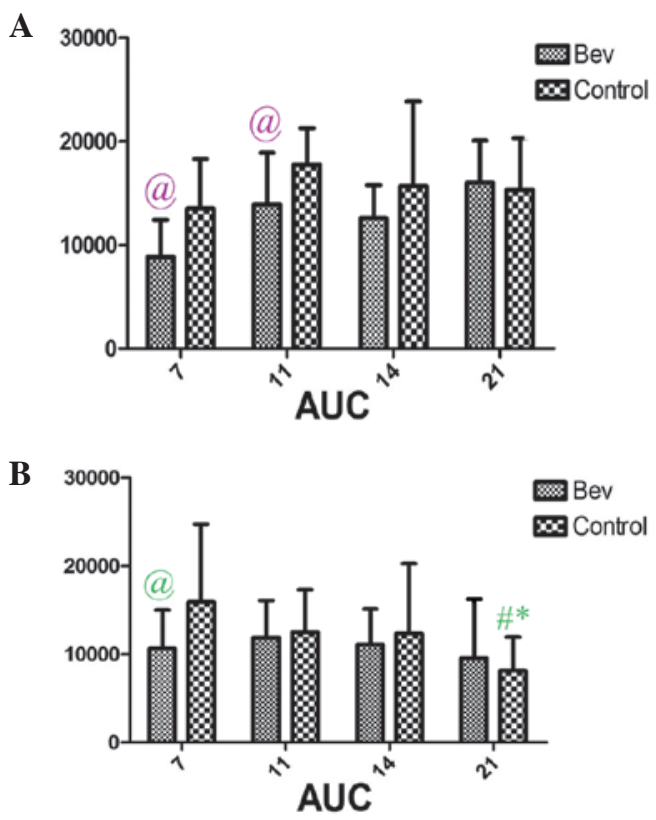

Figure 6. Area under the whole curve (AUC) in the Bev and control groups for (A) $\mathrm{ROI}_{\text {small }}$ and (B) ROI $\mathrm{I}_{\text {total }}$. AUC in the control group was initially increased and then decreased in $\mathrm{ROI}_{\text {small }}$, while it was constantly decreased in $\mathrm{ROI}_{\text {total }}$ with increasing time. For ROI small , AUC in the Bev group was smaller compared with the control group on days 7 and 11 . For ROI total $_{1}$, AUC in the Bev group was smaller compared with the control group on day 7. " $\mathrm{P}<0.05 \mathrm{com}$ pared with AUC on day $7 ;{ }^{*} \mathrm{P}<0.05$ compared with AUC on day $11 ;{ }^{\circledR} \mathrm{P}<0.05$ compared with AUC in the control group. Bev, bevacizumab-treated; ROI, region of interest; $\mathrm{ROI}_{\text {total }}$, includes the whole tumor; $\mathrm{ROI}_{\text {small }}$, includes the tumor area with the highest Imax; Imax, maximum intensity. 
A

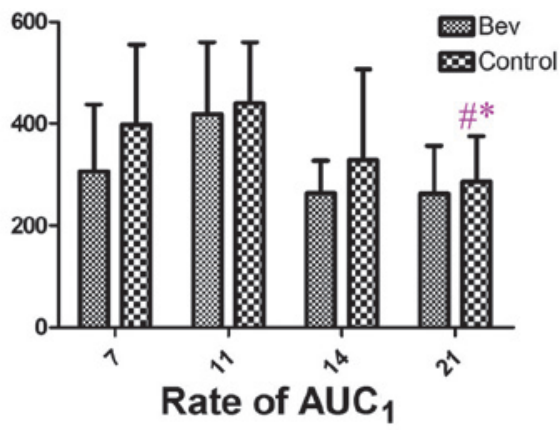

C

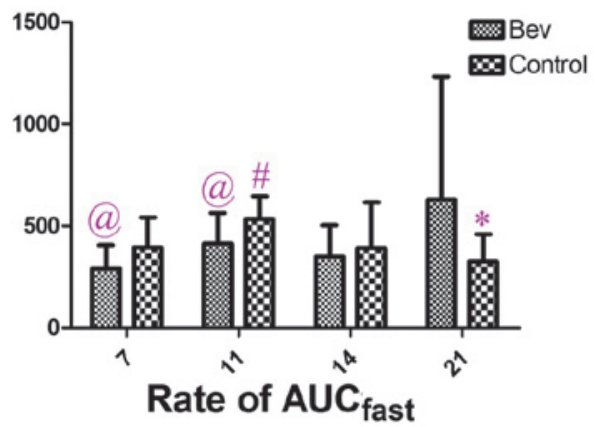

B

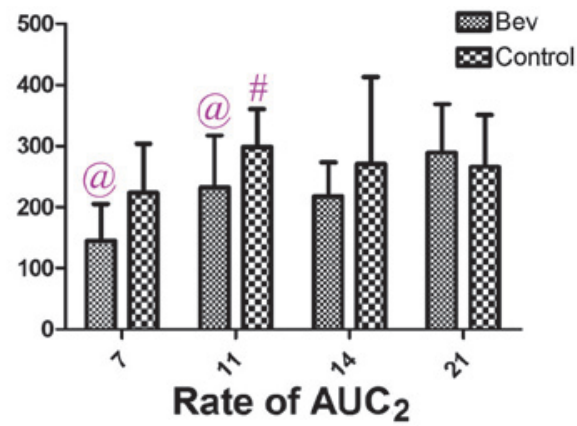

D



Figure 7. Rates of AUC parameters in the Bev and control groups for $\mathrm{ROI}_{\text {small }}$. The rates of $\mathrm{AUC}_{1}, \mathrm{AUC}_{2}, \mathrm{AUC}_{\text {fast }}$ and $\mathrm{AUC}_{\text {slow }}$ in the control group were initially increased and then decreased in $\mathrm{ROI}_{\text {small }}$ with increasing time. In $\mathrm{ROI}_{\text {small }}$, the rates of $\mathrm{AUC}_{2}, \mathrm{AUC}_{\text {fast }}$ and $\mathrm{AUC}$ slow in the Bev group were smaller compared with those in the control group on days 7 and 11. Bev, bevacizumab-treated; ROI, region of interest; $\mathrm{ROI}_{\text {small }}$, includes the tumor area with the highest Imax; Imax, maximum intensity; rate of $\mathrm{AUC}_{1}$, area under the ascending curve $\left(\mathrm{AUC}_{1}\right) / \mathrm{TTP}$; rate of $\mathrm{AUC} \mathrm{C}_{2}$, area under the descending curve $\left(\mathrm{AUC}_{2}=\mathrm{AUC}_{\text {fast }}+\mathrm{AUC}_{\text {slow }}\right) / 60-\mathrm{TTP}$; rate of $\mathrm{AUC}_{\text {fast }}$, area under the fast descending curve $\left(\mathrm{AUC}_{\text {fast }}\right) /(\mathrm{T}-\mathrm{TTP})$; rate of $\mathrm{AUC}$ slow, area under the slow descending curve $\left(\mathrm{AUC}_{\text {slow }}\right) /(60-\mathrm{T})$; TTP, time to peak.

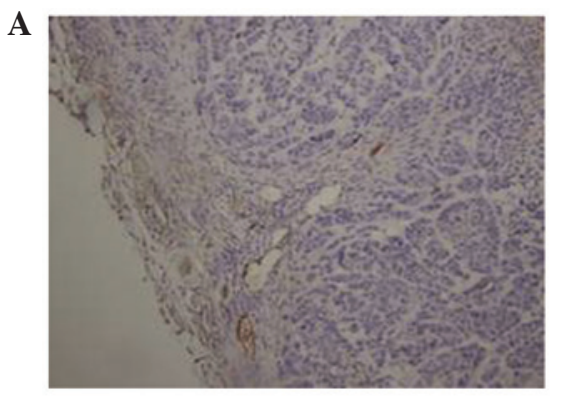

$\mathbf{C}$

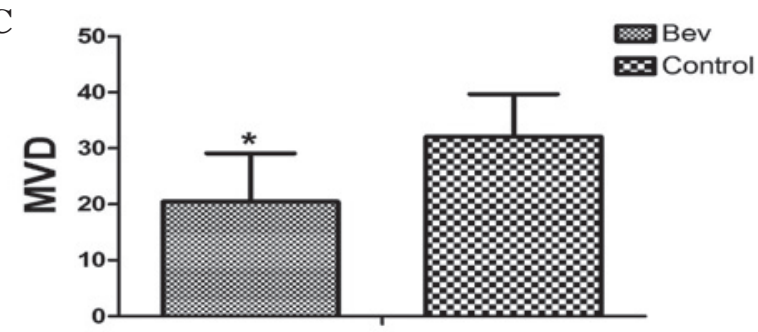

Figure 8. Microvessel density (MVD) in the Bev and control groups. MVD in the (A) Bev and (B) control groups (CD34 staining; magnification, $\mathrm{x} 400$ ); (C) comparison of MVD between the Bev and control groups. " $\mathrm{P}<0.05$ compared with the control group. Bev, bevacizumab-treated.

$\mathrm{V}$ measured by conventional ultrasound increased with increasing time; however, no significant differences were observed between the Bev and control groups at all the four time-points ( $\mathrm{P}>0.05$; Fig. 3 ).

\section{Quantitative analysis of CEUS}

TTP. TTP increased in the control group with increasing time for both $\mathrm{ROI}_{\text {small }}$ and $\mathrm{ROI}_{\text {total }}$. However, no significant

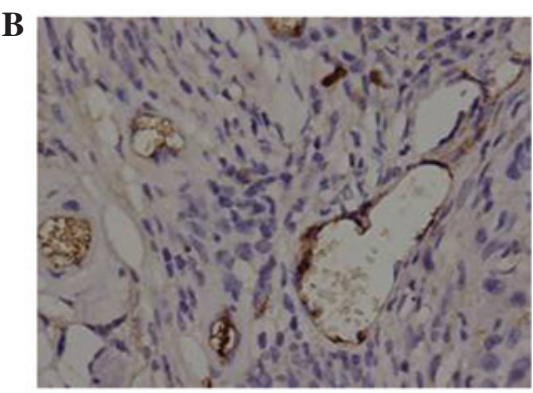

difference in TTP was observed between the Bev and control groups at all the four time-points for both $\mathrm{ROI}_{\text {small }}$ and $\mathrm{ROI}_{\text {total }}$ ( $\mathrm{P}>0.05$; Fig. 4).

Imax. Imax in the control group was initially increased and then decreased in $\mathrm{ROI}_{\text {small }}$ with increasing time, while it was constantly decreased in $\mathrm{ROI}_{\text {total }}$. No significant difference in Imax was observed between the Bev and control groups at all the four time-points for both $\mathrm{ROI}_{\text {small }}$ and $\mathrm{ROI}_{\text {total }}(\mathrm{P}>0.05$; Fig. 5). 
AUC parameters. AUC in the control group was initially increased and then decreased in $\mathrm{ROI}_{\text {small }}$ with increasing time, while it was constantly decreased in $\mathrm{ROI}_{\text {total }}$ (Fig. 6).

For $\mathrm{ROI}_{\text {small }}, \mathrm{AUC}$ and the rates of $\mathrm{AUC}_{2}, \mathrm{AUC}_{\text {fast }}$ and $\mathrm{AUC}_{\text {slow }}$ were smaller in the Bev group compared with those in the control group on days 7 and $11(\mathrm{P}<0.05$; Figs. 6 and 7$)$. For $\mathrm{ROI}_{\text {total }}, \mathrm{AUC}$ and the rates of $\mathrm{AUC}_{2}$ and $\mathrm{AUC}_{\text {slow }}$ were smaller in the Bev group compared with those in the control group on day $7(\mathrm{P}<0.05)$. The remaining parameters were not significantly different between the Bev and control groups $(\mathrm{P}>0.05)$.

Histological examination. AUC in the control group was negatively correlated with MVD $(\mathrm{P}<0.05)$, while no statistical correlation between the remaining CEUS parameters and MVD was observed. MVD was $6.41 \pm 1.52$ in the control group and $4.09 \pm 1.73$ in the Bev group. MVD in the Bev group was significantly lower compared with the control group ( $\mathrm{P}=0.001$; Fig. 8).

\section{Discussion}

Bevacizumab, the humanized anti-VEGF monoclonal antibody, demonstrates a greater binding affinity for human VEGF than murine VEGF-A $(16,17)$. In the present study, we used bevacizumab to treat murine tumors derived from murine colon carcinoma cells instead of human cancer cells. Bevacizumab treatment is less effective in murine tumors, and advanced imaging technology is required to evaluate the effect of bevacizumab treatment on murine tumors. Results of the present study confirmed the anti-angiogenic effect of bevacizumab on murine tumors, since MVD in the Bev group was significantly lower compared with that in the control group. A number of CEUS parameters were also shown to be significantly different between the Bev and control groups and may be used to evaluate the anti-angiogenic effect of bevacizumab.

Studies by Ignee et al (10) and Huang-Wei et al (11) investigated whether ROI alone affected results from the quantitative analysis of CEUS and provided inconsistent conclusions. This may have been due to homogeneously enhanced liver parenchyma in Ignee et al's study, while hepatic focal nodular hyperplasia in Huang-Wei et al's study was inhomogeneously enhanced. In the present study, two ROIs were drawn for each tumor; $\mathrm{ROI}_{\text {total }}$, which included the whole tumor, and $\mathrm{ROI}_{\text {small }}$, which included the most enhanced part of the tumor. The results showed that TTP increased with increasing time in the control group for both $\mathrm{ROI}_{\text {small }}$ and $\mathrm{ROI}_{\text {total }}$. However, Imax and AUC parameters in the control group were characterized by different patterns in $\mathrm{ROI}_{\text {small }}$ and $\mathrm{ROI}_{\text {total }}$; Imax and AUC parameters were initially increased and then decreased in $\mathrm{ROI}_{\text {small }}$, while they were constantly decreased in $\mathrm{ROI}_{\text {total }}$. Furthermore, a greater number of quantitative parameters were significantly different between the Bev and control groups in $\mathrm{ROI}_{\text {small }}$ than in $\mathrm{ROI}_{\text {total }}$. These results suggest that $\mathrm{ROI}_{\text {small }}$ may be more sensitive than $\mathrm{ROI}_{\text {total }}$ for reflecting changes in angiogenesis and blood supply in tumors following anti-angiogenesis treatment. The sensitivity of parameters for the quantitative analysis of CEUS may have been affected by the intratumoral necrotic area, which had no microbubbles in it. Additionally, subcutaneous vessels near the tumor may have affected the sensitivity. Since ultrasound was unable to differentiate between intratumoral vessels and subcutaneous vessels near the tumor (18), and considering the effects of breath movement, subcutaneous vessels near the tumor were more likely to be included in the $\mathrm{ROI}_{\text {total }}$, leading to an inaccurate quantitative analysis of the tumor blood supply.

TTP and Imax are the most commonly used quantitative parameters for CEUS $(9,19,20)$. In the present study, these two parameters were not found to be significantly different between the Bev and control groups, indicating that they were not effective for the evaluation of the anti-angiogenic effect of bevacizumab. Therefore, more effective parameters are required to evaluate the treatment outcomes. AUC parameters, which are considered to be an integration of intensity and time, are being increasingly used (21). In the present study, AUC was defined as the area under the raw curve for $60 \mathrm{sec}$, rather than AUC of the fitting curve for infinite time (9), since the fitting curve may not accurately reflect the raw curve. To avoid the effects of time, $\mathrm{AUC}_{1}, \mathrm{AUC}_{2}, \mathrm{AUC}_{\text {fast }}$ and $\mathrm{AUC}_{\text {slow }}$ were divided by the respective times to determine the rate of each AUC. Our results showed that AUC parameters effectively evaluated the effect of bevacizumab treatment, since $\mathrm{AUC}$ and the rates of $\mathrm{AUC}_{2}$, $\mathrm{AUC}_{\text {fast }}$ and $\mathrm{AUC}_{\text {slow }}$ in the Bev group were decreased compared with those in the control group on days 7 and 11 following tumor establishment. This indicated that AUC parameters were able to reflect early changes in blood supply following bevacizumab treatment. Furthermore, the descending curve may be more important in the evaluation of blood supply change than the ascending curve, since AUC parameters of the descending curve were significantly different between the Bev and control groups, whereas $\mathrm{AUC}_{1}$, the AUC parameter of the ascending curve, was not significantly different between the two groups. However, on days 14 and 21, none of the AUC parameters were significantly different between the two groups. Furthermore, a number of AUC parameters were increased in the Bev group compared with the control group; further investigation is required to interpret these results.

In conclusion, the quantitative analysis of CEUS was demonstrated to be a useful tool to evaluate the anti-angiogenic effect of bevacizumab treatment. $\mathrm{ROI}_{\text {small }}$ was more sensitive compared with $\mathrm{ROI}_{\text {total }}$ for reflecting changes in angiogenesis and blood supply in tumors. AUC parameters were also effective for the evaluation of changes in tumor angiogenesis following bevacizumab treatment.

\section{Acknowledgements}

This study was supported by grants from the National Natural Science Foundation of China (81201100) and the Medical Engineering Cross Foundation of Shanghai Jiaotong University (YG2011MS53).

\section{References}

1. Nicosia RF: What is the role of vascular endothelial growth factor-related molecules in tumor angiogenesis? Am J Pathol 153: 11-16, 1998

2. Dvorak HF, Brown LF, Detmar M and Dvorak AM: Vascular permeability factor/vascular endothelial growth factor, microvascular hyperpermeability, and angiogenesis. Am J Pathol 146: 1029-1039, 1995.

3. Wedam SB, Low JA, Yang SX, et al: Antiangiogenic and antitumor effects of bevacizumab in patients with inflammatory and locally advanced breast cancer. J Clin Oncol 24: 769-777, 2006. 
4. Hochster HS, Hart LL, Ramanathan RK, et al: Safety and efficacy of oxaliplatin and fluoropyrimidine regimens with or without bevacizumab as first-line treatment of metastatic colorectal cancer: results of the TREE Study. J Clin Oncol 26: 3523-3529, 2008.

5. Quaia E: Assessment of tissue perfusion by contrast-enhanced ultrasound. Eur Radiol 21: 604-615, 2011.

6. Shiyan L, Pintong H, Zongmin W, Fuguang H, Zhiqiang Z, Yan Y and Cosqrove D: The relationship between enhanced intensity and microvessel density of gastric carcinoma using double contrast-enhanced ultrasonography. Ultrasound Med Biol 35: 1086-1091, 2009.

7. Lassau N, Koscielny S, Albiges L, et al: Metastatic renal cell carcinoma treated with sunitinib: early evaluation of treatment response using dynamic contrast-enhanced ultrasonography. Clin Cancer Res 16: 1216-1225, 2010.

8. Lassau N, Koscielny S, Chami L, et al : Advanced hepatocellular carcinoma: early evaluation of response to bevacizumab therapy at dynamic contrast-enhanced US with quantification - preliminary results. Radiology 258: 291-300, 2011.

9. Zhou JH, Zheng W, Cao LH, et al: Quantitative evaluation of viable tissue perfusion changes with contrast-enhanced greyscale ultrasound in a mouse hepatoma model following treatment with different doses of thalidomide. Br J Radiol 84: 826-832, 2011.

10. Ignee A, Jedrejczyk M, Schuessler G, Jakubowski W and Dietrich CF: Quantitative contrast enhanced ultrasound of the liver for time intensity curves - reliability and potential sources of errors. Eur J Radiol 73: 153-158, 2010.

11. Huang-Wei C, Bleuzen A, Bourlier P, Roumy J, Bouakaz A, Pourcelot L and Tranquart F: Differential diagnosis of focal nodular hyperplasia with quantitative parametric analysis in contrast-enhanced sonography. Invest Radiol 41: 363-368, 2006.

12. Zhang HP and Du LF: Quantitative analysis of contrast-enhanced ultrasound and its clinical value. Chin J Med Imaging Technol 27: 1713-1716, 2011.
13. Schwarz KQ, Chen X, Bezante GP, Phillips D and Schlief R: The Doppler kinetics of microbubble echo contrast. Ultrasound Med Biol 22: 453-462, 1996.

14. Kämmerer U, Kapp M, Gassel AM, et al: A new rapid immunohistochemical staining technique using the EnVision antibody complex. J Histochem Cytochem 49: 623-630, 2001.

15. Weidner N: Current pathologic methods for measuring intratumoral microvessel density within breast carcinoma and other solid tumors. Breast Cancer Res Treat 36: 169-180, 1995.

16. Bock F, Onderka J, Dietrich T, et al: Bevacizumab as a potent inhibitor of inflammatory corneal angiogenesis and lymphangionesis. Invest Ophthalmol Vis Sci 48: 2545-2552, 2007.

17. Hashemian MN, Moghimi S, Kiumehr S, Riazi M and Amoli FA: Prevention and treatment of corneal neovascularization: comparison of different doses of subconjunctival bevacizumab with cortisteroid in experimental rats. Ophthalmic Res 42: 90-95, 2009.

18. Krix M, Kiessling F, Vosseler S, et al: Sensitive noninvasive monitoring of tumor perfusion during antiangiogenic therapy by intermittent bolus-contrast power Doppler sonography. Cancer Res 63: 8264-8270, 2003.

19. Broumas AR, Pollard RE, Bloch SH, Wisner ER, Griffey SD and Ferrara KW: Contrast-enhanced computed tomography and ultrasound for the evaluation of tumor blood flow. Invest Radiol 40: 134-147, 2005

20. McCarville MB, Streck CJ, Dickson PV, Li CS, Nathwani AC and Davidoff AM: Angiogenesis inhibitors in a murine neuroblastoma model: quantitative assessment of intratumoral blood flow with contrast-enhanced gray-scale US. Radiology 240: 73-81, 2006.

21. Seidel G, Algermissen G, Christopg A, et al: Harmonic imaging of the human brain. Visualization of brain perfusion with ultrasound. Stroke 31: 151-154, 2000. 